\title{
BMJ Open Seroprevalence study of SARS-CoV-2 antibodies in healthcare workers following the first wave of the COVID-19 pandemic in a tertiary-level hospital in the south of Ireland
}

To cite: Faller E, Wyse A, Barry R, et al. Seroprevalence study of SARS-CoV-2 antibodies in healthcare workers following the first wave of the COVID-19 pandemic in a tertiary-level hospital in the south of Ireland. BMJ Open 2021;11:e051415. doi:10.1136/ bmjopen-2021-051415

- Prepublication history and additional supplemental material for this paper are available online. To view these files, please visit the journal online (http://dx.doi.org/10.1136/ bmjopen-2021-051415).

Received 18 March 2021 Accepted 24 May 2021

Check for updates

(c) Author(s) (or their employer(s)) 2021. Re-use permitted under CC BY-NC. No commercial re-use. See rights and permissions. Published by BMJ.

For numbered affiliations see end of article.

Correspondence to

Dr Eamonn Faller;

eamonnfaller@gmail.com

\section{ABSTRACT}

Objective This study investigated seroprevalence of SARS-CoV-2-specific IgG antibodies, using the Abbott antinucleocapsid IgG chemiluminescent microparticle immunoassay (CMIA) assay, in five prespecified healthcare worker (HCW) subgroups following the first wave of the COVID-19 pandemic.

Setting An 800-bed tertiary-level teaching hospital in the south of Ireland.

Participants Serum was collected for anti-SARS-CoV-2 nucleocapsid IgG using the Abbott ARCHITECT SARS-CoV-2 IgG CMIA qualitative assay, as per the manufacturer's specifications.

The groups were as follows: (1) HCWs who had real-time PCR (RT-PCR) confirmed COVID-19 infection (>1-month postpositive RT-PCR); (2) HCWs identified as close contacts of persons with COVID-19 infection and who subsequently developed symptoms (virus not detected by RT-PCR on oropharyngeal/nasopharyngeal swab); (3) HCWs identified as close contacts of COVID-19 cases and who remained asymptomatic (not screened by RT-PCR); (4) HCWs not included in the aforementioned groups working in areas determined as high-risk clinical areas; and (5) HCWs not included in the aforementioned groups working in areas determined as low-risk clinical areas.

Results Six of 404 (1.49\%) HCWs not previously diagnosed with SARS-CoV-2 infection (groups 2-5) were seropositive for SARS-CoV-2 at the time of recruitment into the study.

Out of the 99 participants in group 1, 72 had detectable IgG to SARS-CoV-2 on laboratory testing (73\%). Antibody positivity correlated with shorter length of time between RT-PCR positivity and antibody testing. Quantification cycle value on RT-PCR was not found to be correlated with antibody positivity.

Conclusions Seroprevalence of SARS-CoV-2 antibodies in HCWs who had not previously tested RT-PCR positive for COVID-19 was low compared with similar studies.

\section{Strengths and limitations of this study}

We successfully recruited the numbers that we had aimed for in each of the prespecified groups.

- This was a single-centre study in an area of relatively low SARS-CoV-2 prevalence.

- Enrolment began 8 weeks after peak regional prevalence, and therefore, IgG antibodies may have become undetectable in a proportion of participants.

- Recruitment of groups 3-5 was by self-selection and therefore was not a true random sample of these groups

- Quantification cycle $\left(\mathrm{C}_{\mathrm{q}}\right)$ values were only available for 69 of the 99 participants who were real-time PCR positive, including only 12 of whom were IgG negative. It is therefore difficult to draw any firm conclusion as regards the correlation between $\mathrm{C}_{q}$ value and antibody positivity.

\section{INTRODUCTION}

Healthcare workers (HCWs) at the front line treating patients with suspected or confirmed COVID-19 have been heavily impacted by the pandemic. Due to potential occupational exposures, HCWs are at higher risk of infection from patients or from other HCWs than the general population. In a study published in July 2020, there was an estimated HR of 3.40 for COVID-19 infection in HCWs compared with risk of infection in the general population. ${ }^{1}$ Indeed, as of November 2020 in Ireland, the Health Protection and Surveillance Centre put the number of HCW infections at 10976 , accounting for $16.6 \%$ of total infections. ${ }^{2}$ 
The first case of SARS-CoV-2 infection was reported in Ireland on 29 February 2020 relating to travel. On 5 March, a patient was diagnosed with SARS-CoV-2 infection who had been ventilated in the intensive care unit of Cork University Hospital (CUH) with atypical pneumonia despite having no epidemiological link to a known case or area of high prevalence. This was the first documented community acquisition of SARS-CoV-2 in Ireland and was an indication of potential widespread community transmission. ${ }^{3}$ From this date, additional infection prevention measures were instituted in $\mathrm{CUH}$, including testing and contact tracing of all symptomatic patients and staff, changes in hospital operations and provision of personal protective equipment (PPE).

Seroprevalence studies can provide relevant information on the proportion of a population who have experienced a recent or past infection. Monitoring the prevalence of infection among HCWs is useful for assessing the level of exposure and identifying high-risk areas.

There have been a number of studies that have attempted to characterise the immunological response to COVID-19. Median time to seroconversion is estimated at 9-12 days following onset of symptoms depending on the antibody measured, with up to $100 \%$ developing antibodies by day $21 .{ }^{4}$ Sensitivity of assays measuring the antinucleocapsid antibodies has been shown to decline from 60 days following PCR positivity. ${ }^{5}$ However, correlation between seropositivity or antibody levels and protection against reinfection remains to be fully determined. ${ }^{67}$

The aim of this study was to investigate seroprevalence of SARS-CoV-2-specific IgG antibodies, using the Abbott antinucleocapsid IgG chemiluminescent microparticle immunoassay (CMIA), in five prespecified HCW subgroups following the first surge of the pandemic in a region of relative low prevalence of COVID-19 infection.

\section{METHODS}

\section{Study design and participants}

This study was undertaken over a 6 -week period from the 27 May 2020 to 7 July 2020 in CUH, an 800-bed university teaching hospital. CUH is the tertiary referral centre in the South West of Ireland serving a population of 1.1 million people. The study was designed to recruit 100 HCWs from five prespecified subgroups as outlined as follows.

\section{HCW subgroups}

1. HCWs who had real-time PCR (RT-PCR) confirmed COVID-19 infection ( $>1$-month postpositive RT-PCR).

2. HCWs identified as close contacts of persons with COVID-19 infection and who subsequently developed symptoms (virus not detected by RT-PCR on oropharyngeal/nasopharyngeal swab).

3. HCWs identified as close contacts of COVID-19 cases and who remained asymptomatic (not screened by RTPCR).
4. HCWs not included in the aforementioned groups working in areas determined as high-risk clinical areas.

5. HCWs not included in the aforementioned groups working in areas determined as low-risk clinical areas.

Basic demographic data including age, gender, occupation and comorbid illness were collected by means of a self-administered questionnaire (online supplemental appendix 1).

HCWs from groups 1 (previous confirmed RT-PCR COVID-19 infection) and group 2 (close contact of COVID-19 case with virus not detected by RT-PCR on oropharyngeal/nasopharyngeal swab when symptomatic) were contacted by the occupational health department. As there were fewer than 100 HCWs with RT-PCR-confirmed COVID-19 in CUH, HCWs with RT-PCR-confirmed COVID-19 from affiliated regional centres were invited to participate.

HCWs from group 3-5 were recruited by open invitation, and group allocation was confirmed by recruiting investigators.

\section{Inclusion criteria}

HCWs aged 18 years or over, fluent in English working in CUH or affiliated centres in the region were eligible to participate. HCWs were defined as those who deliver care and services to patients, either directly as physicians or nurses, healthcare attendants, or other support staff (porters, administrative officers, cleaning, maintenance, etc).

\section{Exclusion criteria}

HCWs who tested positive by RT-PCR for SARS-CoV-2 within 30 days of recruitment to the study or reporting symptoms of COVID-19 at time of recruitment were deemed ineligible to participate. However, there were no diagnosed infections among staff in our institution in the 30 days prior to enrolment.

\section{Patient and public involvement}

Patients and the public were not involved in the design of this study; however, feedback was enlisted on the sampling procedures and appropriateness of sampling modalities that the researchers used as part of the study (venepuncture for antinucleocapsid antigen as well as saliva and point of care testing used in the validation of other testing modalities not included in this paper).

\section{Laboratory procedures \\ Serological testing}

Serum was collected for anti-SARS-CoV-2 nucleocapsid IgG using the Abbott ARCHITECT SARS-CoV-2 IgG CMIA qualitative assay, as per the manufacturer's specifications. The Abbott Elisa Kit (Abbott Diagnostics) uses a nucleocapsid protein as the antigen and reported a $100 \%$ concordance (95\% CI $95.89 \%$ to $100 \%$ ) with their RT-PCR positive panel $>14$ days after symptom onset and 99.6\% negative on their historical pre-COVID-19 controls $(95 \% \text { CI } 98.98 \% \text { to } 99.89 \%)^{8}$ 


\section{qRT-PCR for SARS-CoV-2}

HCWs from group 1 and group 2 who had close contact with a case of COVID-19 infection and developed symptoms had a combined oropharyngeal/nasopharyngeal swab undertaken as part of clinical care. Laboratory confirmation of SARS-CoV-2 infection was performed using the MagNA Pure 24/MagNA Pure LC (Roche Diagnostics) extraction system and Realstar (Altona Diagnostics, Hamburg, Germany) or EURORealTime (EUROIMMUN, Lübeck, Germany) SARS-CoV-2 qRT-PCR kits, as per the manufacturer's instructions. Target detection was reported on a LightCycler 480 Instrument II (Roche) if the quantification cycle $\left(\mathrm{C}_{\mathrm{q}}\right)$ value was $<40$. In the absence of assay standardisation with RNA copy number controls, the $\mathrm{C}_{\mathrm{q}}$ value was used as a relative quantitative indication of viral load.

\section{Statistical analysis}

SPSS V.26.0 and GraphPad Prism V.8 were used for statistical analysis. $\chi^{2}$ test was used to compare categorical variables. Independent samples t-test was used to compare means of independent scale variables where frequencies were normally distributed and Mann-Whitney U test was used to compare continuous variables where frequencies were non-normally distributed. Results were deemed to be significant if the $p$ value is $<0.05$.

\section{RESULTS}

\section{Sample characteristics}

Of 4500 staff employed directly in CUH, 503 HCWs were recruited to the study. Baseline demographics of participants are outlined in table 1.

The age range of participants was 20-65 years (IQR $30-47$ years), and $77 \%$ were female. There were no significant between-group differences in age profiles. Nurses were the most represented professional group (41.7\%) followed by doctors $(35.0 \%)$.

Overall level of comorbidity was low across the groups, with $58.8 \%$ of the study population reporting no known/ current medical issues. There were a significantly greater number of ex-smokers among participants in group 1 compared with other groups $(\mathrm{p}<0.001)$ and a significantly greater number of current smokers in group $2(\mathrm{p}=0.021)$. There was no significant between-group difference for any of the other comorbidities listed.

Of the participants, $187(187 / 503,37.2 \%)$ worked in high-risk settings. These were deemed to be areas in which HCWs were having daily contact with patients with confirmed or suspected COVID-19 infection during the peak of the local epidemic.

Of the participants, $469(469 / 503,93.2 \%)$ were working in $\mathrm{CUH}$, the institution in which the study was conducted with 34 participants (all from group 1) recruited from affiliated institutions within the South/ Southwest Hospital Group.

\section{Seroprevalence}

Overall, 78 of $503(15.5 \%)$ HCWs who participated in the study were seropositive for SARS-CoV-2 at time of recruitment into the study. Table 2 presents serology results by the HCW group.

Out of the 99 participants in group 1, 72 had detectable IgG to SARS-CoV-2 on laboratory testing (73\%). Longitudinal IgG detection from date of positive RT-PCR is displayed in figure 1 . The mean period of time from RT-PCR positivity to IgG testing was significantly shorter in the IgG-positive group, with a mean of 69.3 days compared with 77.0 days in those who were antibody negative $(p=0.025)$. There was no correlation noted between antibody seropositivity and age $(\mathrm{p}=0.63)$, gender $(\mathrm{p}=0.416)$ or presence of one or more comorbidities $(\mathrm{p}=0.935)$.

Only 1 of 99 HCWs with RT-PCR-confirmed COVID-19 required hospitalisation for management of infection, with the vast majority experiencing mild symptoms.

RT-PCR $\mathrm{C}_{\mathrm{q}}$ values were available for 69 of the participants in group 1. This included 57 participants who were $\operatorname{IgG}$ positive and 12 who were IgG negative. There was no correlation found between RT-PCR $\mathrm{C}_{\mathrm{q}}$ values and SARS-CoV-2 IgG detection ( $\mathrm{p}=0.943)$.

Overall seroprevalence was low among groups $2-5$, with IgG antibodies detected in only 6 out of 404 participants $(1.49 \%)$. Prevalence was comparable between the four groups with $\operatorname{IgG}$ antibodies detected in two participants in group $2(1.9 \%)$, one in group $3(1.1 \%)$, one in group $4(1.0 \%)$ and two in group $5(1.9 \%)$.

\section{DISCUSSION}

Of 99 HCWs with RT-PCR-confirmed SARS-CoV-2 infection, 73\% (72) had detectable antinucleocapsid IgG antibodies to SARS-CoV-2. A single factor, time interval from positive RT-PCR was associated with antibody detection. This is consistent with much of the wider literature in indicating that antinucleocapsid IgG antibodies to SARS-CoV-2 begin to decline from day 60 following positive PCR, particularly in individuals with mild or asymptomatic primary infection. ${ }^{7910}$ Although a higher sensitivity has been reported for this assay, ${ }^{11}$ our data indicate that sensitivity drops over time, potentially limiting usefulness of this assay over the longer term.

We report a seroprevalence of SARS-CoV-2 IgG in HCWs in our institution not previously diagnosed with COVID-19 by RT-PCR of $1.49 \%$. The (SCOPI) conducted over the same period estimated overall seroprevalence in the general population at $1.7 \%,{ }^{12}$ with regional differences between urban Dublin $(3.1 \%)$ and rural Sligo $(0.6 \%)$. In Cork and Kerry, the two main counties served by our hospital, HCW infections represented $23 \%$ of total infections during the first wave. This was a smaller percentage than the figure seen nationally of $32.1 \%$ and would indicate that there was a lower proportion of HCW infected in Cork. ${ }^{13}$

Seroprevalence in HCWs without previously diagnosed COVID-19 is lower than in the majority of published international studies that report seroprevalence among 
Open access

Table 1 Participant demographics and comorbidities

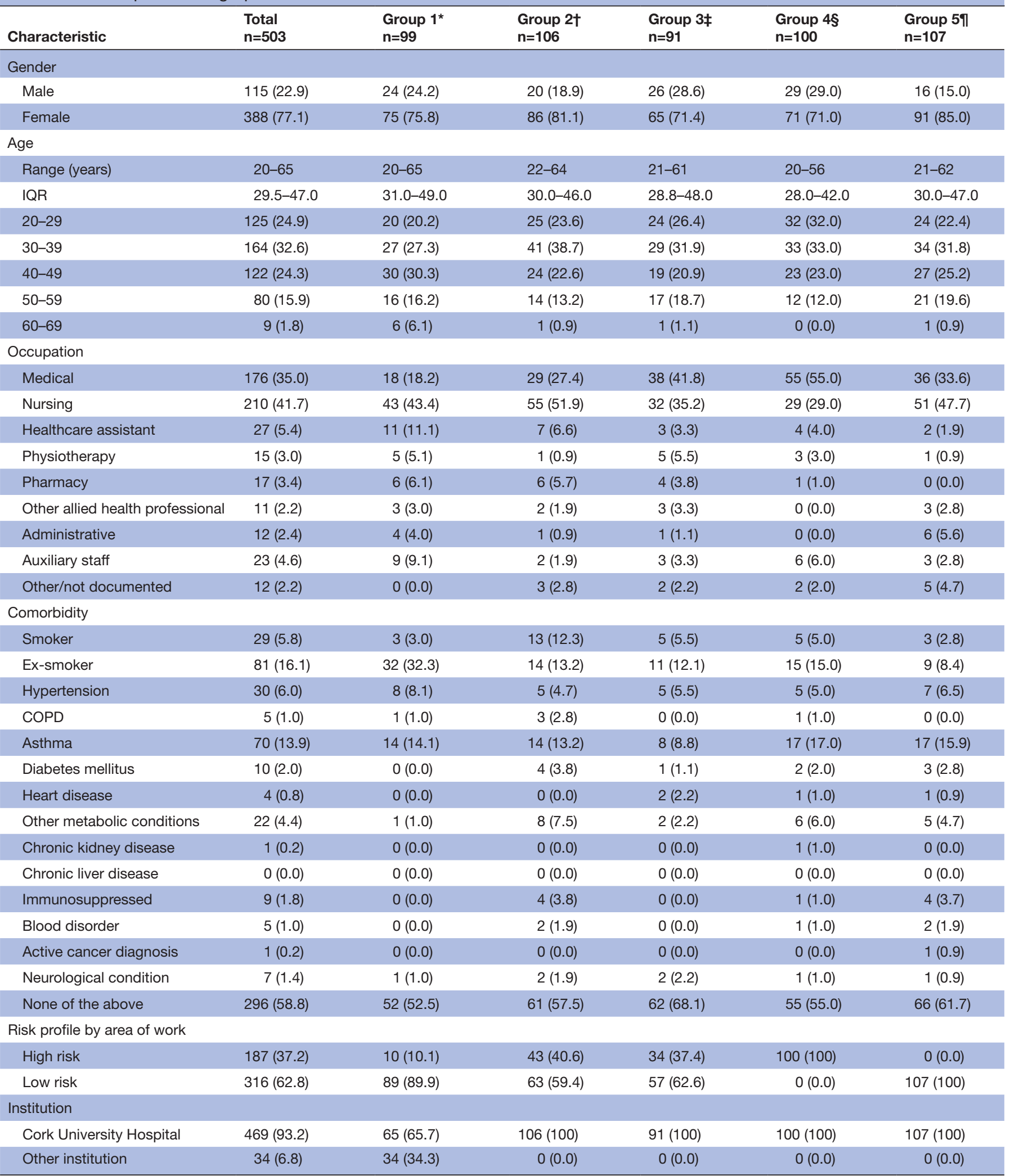

Data are presented as $\mathrm{n}$ (\% of total displayed at top of individual columns) unless otherwise stated.

${ }^{*}$ RT-PCR-confirmed COVID-19 infection (>1-month postpositive RT-PCR).

†Close contacts of persons with COVID-19 infection and who subsequently developed symptoms (virus not detected by RT-PCR on oro/nasopharyngeal swab).

$\neq$ Close contacts of COVID-19 cases and who remained asymptomatic.

§CWs working in areas determined as high-risk clinical areas.

ๆHCWs working in areas determined as low-risk clinical areas.

COPD, chronic obstructive pulmonary disease; RT-PCR, real-time PCR. 
Table 2 SARS-CoV-2 IgG seropositivity by study group

\begin{tabular}{lcc}
\hline Study group & Total & IgG positive \\
\hline Group 1* & 99 & $72(72.7)$ \\
Group 2† & 106 & $2(1.9)$ \\
Group 3‡ & 91 & $1(1.1)$ \\
Group 4§ & 100 & $1(1.0)$ \\
Group 5ๆ & 107 & $2(1.9)$ \\
Total & 503 & $78(15.5)$ \\
\hline
\end{tabular}

Data are presented as $\mathrm{n}(\%)$ or total in first column.

${ }^{*}$ RT-PCR-confirmed COVID-19 infection (>1 month postpositive RTPCR).

†Close contacts of persons with COVID-19 infection and who subsequently developed symptoms (virus not detected by RT-PCR on oropharyngeal/nasopharyngeal swab).

$\ddagger$ Close contacts of COVID-19 cases and who remained asymptomatic. $\S \mathrm{HCW}$ working in areas determined as high-risk clinical areas. IHCWs working in areas determined as low-risk clinical areas. HCW, healthcare worker; RT-PCR, real-time PCR.

HCWs not previously diagnosed with COVID-19 (groups 2-5) of anywhere between $1.6 \%$ and $9.0 \% .^{14-19}$

In the USA, a study of a multistate hospital network reported $6 \%$ seropositivity in 3248 HCWs across 13 geographically diverse institutions. Notably, $69 \%$ of those who were antibody-positive did not have a prior diagnosis of COVID-19 infection. ${ }^{15}$ A study of 46117 HCWs in the greater New York City area across 52 sites revealed a $13.7 \%$ total seropositivity to SARS-CoV-2-specific IgG antibodies. $10.3 \%$ of those who had previously tested RT-PCR negative and $9.0 \%$ of those who had never been previously tested were seropositive. ${ }^{20}$ In Madrid, a large tertiarylevel institution reported a seroprevalence of $11.2 \%$ in a random sample of HCWs at the peak of the first wave in Europe (28 March-9 April 2020). Of this cohort, 40.0\% had not had previously diagnosed COVID-19 infection. ${ }^{14}$ However, one smaller-scale study of 316 HCWs in Essen in Germany found just $5(1.6 \%)$ were seropositive, none of whom had previously tested positive. ${ }^{16}$

Longitudinal Ab detection

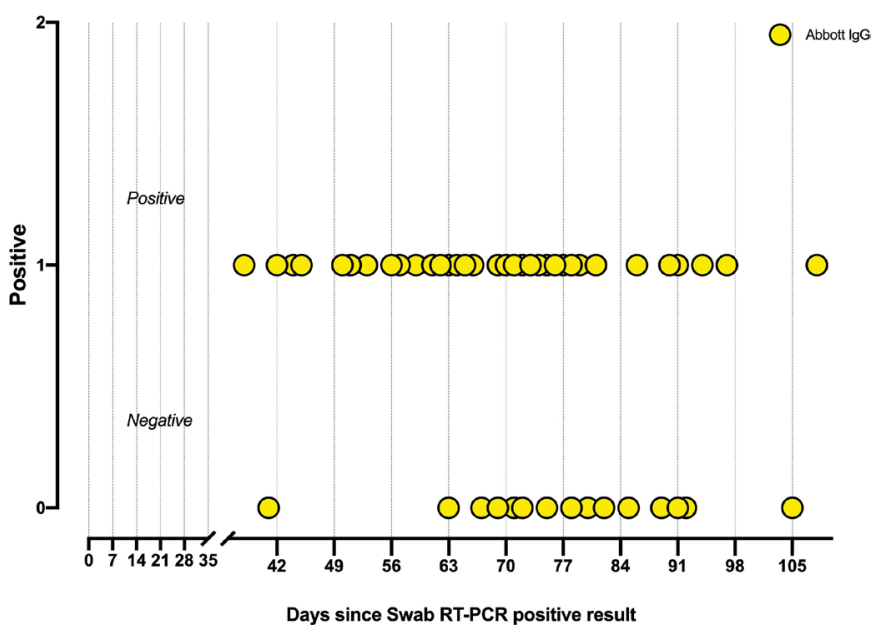

Figure 1 Group one longitudinal SARS-CoV-2 IgG detection since date of positive RT-PCR. $n=99$.
This was particularly surprising given that rate of asymptomatic infection in COVID-19 is thought to be about $15 \% .^{21}$ Only 6 out of 105 participants $(5.7 \%)$ in our study with laboratory evidence of SARS-CoV-2 infection were not diagnosed at time of infection. This was despite guidelines applicable early in the pandemic which dictated that only symptomatic individuals be tested for COVID-19.

There are a number of factors that may have contributed to the low seroprevalence of SARS-CoV-2 IgG in the previously undiagnosed cohort.

The number of patients assessed or hospitalised with COVID-19 $(n=150)$ at our institution was comparatively low during the first wave of the pandemic, and therefore staff may have been exposed to a lower number of COVID-19 patients than in other institutions. The regional prevalence was also comparatively low with a total of 1700 cases reported in Cork as of August 2020 with a peak incidence of 104 cases per 100000 on 27 March 2020. ${ }^{13}$

At no stage during the surge was there an interruption in PPE supply in our institution, and high standards of infection prevention and control were employed throughout. At all times, the guideline-recommended PPE was available to staff for the assessment of COVID-19 confirmed and suspected patients. ${ }^{22}$

Public transport usage by CUH staff is comparatively low, and there is no tram or commuter rail service serving the hospital. This would potentially reduce overall exposure of staff to tightly congregated environments. There are some data to suggest that use of public transport is positively correlated with antibody positivity. ${ }^{23}$

Easily accessible RT-PCR testing and recommendation for quarantine of symptomatic staff members were implemented locally from identification of our first case of COVID-19 on 5 March 2020. This enabled diagnosis of the vast majority of symptomatic infections from the outset with isolation of these cases minimising risk of onward transmission to patients or other HCWs.

Given antibody positivity was only $73 \%$ in group 1 , it is possible that HCWs in groups 2-5 were infected but have had undetectable antibodies at the time of sampling. This would result in a potential underestimate of previously infected individuals in these groups.

As well as within hospitals, similar targeted epidemiological studies would undoubtedly be useful in high-risk, high-prevalence settings such as universities, schools and other healthcare institutions to gain a better understanding of patterns of transmission.

Limitations of this study include its being a singlecentre study undertaken in an area of relatively low prevalence of COVID-19. Enrolment began 8 weeks after peak regional prevalence, and therefore IgG antibodies may have become undetectable in a proportion of participants. $^{24}$ The assay used in the study, Abbott Architect SARS-CoV-2 IgG CMIA, is a qualitative assay, so therefore we were unable to quantify antibody levels in participants. Recruitment of groups 3-5 was by self-selection and therefore was not a true random sample of these groups. 
Data regarding $\mathrm{C}_{\mathrm{q}}$ were only available for 69 participants, of whom only 12 were $\operatorname{IgG}$ negative. Therefore, numbers would not be sufficient to draw a firm conclusion as to the lack of correlation between viral load and subsequent IgG positivity.

\section{CONCLUSION}

In the face of the ongoing COVID-19 pandemic, it is important to define the epidemiology of infection in the healthcare setting. Hospital-wide screening for antibodies to SARS-CoV-2 can profile transmission dynamics and inform infection control and prevention policies. With rollout of effective vaccination on the horizon, studies such as this may inform recommendations for prioritisation of immunisation in the context of potentially limited initial supplies.

It is essential that learning from experience of the initial surge of COVID-19 in the healthcare setting informs future practice and response to optimally protect HCWs and vulnerable patients.

\section{Author affiliations}

${ }^{1}$ Department of Infectious Disease, Cork University Hospital, Cork, Ireland

${ }^{2}$ Department of Microbiology, Cork University Hospital, Cork, Ireland

${ }^{3}$ Department of Paediatrics, Cork University Hospital, Cork, Ireland

${ }^{4}$ Department of Occupational Health, Cork University Hospital Group, Cork, Ireland

${ }^{5}$ School of Medicine, University College Cork, Cork, Cork, Ireland

\section{Twitter Eamonn Faller @eamofaller and Mary Horgan @profmaryhorgan}

Acknowledgements We acknowledge and thank the staff of CUH and affiliated hospitals in the South/Southwest hospital group who participated in this study and also the Health Research Board, Clinical Research Facility, Cork, for the resources and effort contributed towards this study, in particular, Jennifer Connolly, Niamh Kelly, Maeve Kelsey and Lisa McSweeney.

Contributors EF: study concept and design, protocol development; drafted the paper, helped organise logistics of the sample collection. AW: organised and oversaw sample collection for groups 2-5, edited and signed off on the paper. RB: edited and drafted sections of the paper pertaining to microbiological assays. $\mathrm{KC}, \mathrm{CE}, \mathrm{PF}, \mathrm{CF}, \mathrm{EH}, \mathrm{SL}, \mathrm{AM}-\mathrm{B}$, EM and DOS: sample collection, paper edits. GK and GOS: enlisted groups 1 and 2 for participation, paper edits. JAE: edits to the paper. DOS: validated and performed the Abbott assay for all these samples. CD: validated all the SARS-CoV-2 assays listed and personally performed many of the assays from March and April. JB: personally performed many of the assays from March and April. MP: study concept and design, protocol development. Finalised aspects of paper pertaining to microbiology. JG: study concept and design, protocol development; finalised aspects of paper pertaining to occupational health. JM: study concept and design, protocol development, substantial edits and input in all sections of paper. LJF: study concept and design, protocol development and substantial input in all sections of paper. SOR: study concept and design, protocol development, substantial edits and input in all sections of paper. $\mathrm{MH}$ : edited and helped finalise paper. CS: study concept and design, protocol development and substantial edits and input to all sections, finalised the paper. All authors approved the final manuscript.

Funding The study was supported by Cork University Hospital, affiliated institutions in the hospital group and University College Cork.

Competing interests None declared.

Patient consent for publication Not required.

Ethics approval Written informed consent was obtained from healthcare workers using the document contained in the online supplemental appendix 1 . The clinical research ethics committee of the Cork Teaching Hospitals granted ethics approval for this study (ECM 4 (a) 16/06/2020).

Provenance and peer review Not commissioned; externally peer reviewed.
Data availability statement Data are available upon reasonable request. The authors are happy to share data with a data repository if paper is accepted for publication.

Supplemental material This content has been supplied by the author(s). It has not been vetted by BMJ Publishing Group Limited (BMJ) and may not have been peer-reviewed. Any opinions or recommendations discussed are solely those of the author(s) and are not endorsed by BMJ. BMJ disclaims all liability and responsibility arising from any reliance placed on the content. Where the content includes any translated material, BMJ does not warrant the accuracy and reliability of the translations (including but not limited to local regulations, clinical guidelines, terminology, drug names and drug dosages), and is not responsible for any error and/or omissions arising from translation and adaptation or otherwise.

Open access This is an open access article distributed in accordance with the Creative Commons Attribution Non Commercial (CC BY-NC 4.0) license, which permits others to distribute, remix, adapt, build upon this work non-commercially, and license their derivative works on different terms, provided the original work is properly cited, appropriate credit is given, any changes made indicated, and the use is non-commercial. See: http://creativecommons.org/licenses/by-nc/4.0/.

\section{ORCID iDs}

Eamonn Faller http://orcid.org/0000-0002-3873-8508

Corinna Sadlier http://orcid.org/0000-0002-8415-2022

\section{REFERENCES}

1 Nguyen LH, Drew DA, Graham MS, et al. Risk of COVID-19 among front-line health-care workers and the general community: a prospective cohort study. Lancet Public Health 2020;5:e475-83.

2 Health Protection and Surveillance Centre. Report of the profile of COVID-19 cases in healthcare workers in Ireland, 2020.

3 Faller E, Lapthorne S, Barry R. The presentation and diagnosis of the first known community- transmitted case of SARS-CoV-2 in the Republic of Ireland. Ir Med J 2020;113:2-6 http://imj.ie/ the-presentation-and-diagnosis-of-the-first-known-communitytransmitted-case-of-sars-cov-2-in-the-republic-of-ireland/

4 Van Elslande J, Decru B, Jonckheere S, et al. Antibody response against SARS-CoV-2 spike protein and nucleoprotein evaluated by four automated immunoassays and three ELISAs. Clin Microbiol Infect 2020;26:1557.e1-1557.e7.

5 Van Elslande J, André E, Van Ranst M, et al. Immunoassays for anti-SARS-CoV-2 antibodies: recent insights. Lancet Infect Dis 2021;21:e120.

6 Abbasi J. The promise and peril of antibody testing for COVID-19. JAMA 2020;323:1881-1883.

7 Ibarrondo FJ, Fulcher JA, Goodman-Meza D, et al. Rapid decay of Anti-SARS-CoV-2 antibodies in persons with mild Covid-19. N Engl J Med 2020;383:1085-7.

8 Roche. Roche's COVID-19 antibody test receives FDA Emergency Use Authorization and is available in markets accepting the CE mark. Available: https://www.roche.com/dam/jcr:409cf75c-a33a48f8-8a48-c7d9a71fcfab/en/03052020-rochemediareleae-elecsyscovid19.pdf

9 Long Q-X, Tang X-J, Shi Q-L, et al. Clinical and immunological assessment of asymptomatic SARS-CoV-2 infections. Nat Med 2020;26:1200-4.

10 Muecksch F, Wise $\mathrm{H}$, Batchelor B, et al. Longitudinal analysis of clinical serology assay performance and neutralising antibody levels in COVID19 convalescents. medRxiv 2020. doi:10.1101/2020.08.05.2 0169128. [Epub ahead of print: 06 Aug 2020].

11 Bryan A, Pepper G, Wener MH. Performance characteristics of the Abbott architect SARS-CoV-2 IgG assay and seroprevalence testing in Idaho. medRxiv 2020.

12 Health protection and surveillance centre. preliminary report of the results of the study to investigate COVID-19 infection in people living in Ireland (SCOPI): a national seroprevalence study, JuneJuly. Dublin, 2020. Available: https://www.hpsc.ie/a-z/respiratory/ coronavirus/novelcoronavirus/scopi/SCOPI report preliminary results final version.pdf

13 Ni Bhuachalla C, Murphy N, O'Sullivan M. COVID-19: the first 100 days in the South of Ireland. Ir Med J 2020;113:185 http://imj.ie/ covid-19-the-first-100-days-in-the-south-of-ireland/

14 Garcia-Basteiro AL, Moncunill G, Tortajada M, et al. Seroprevalence of antibodies against SARS-CoV-2 among health care workers in a large Spanish reference Hospital. Nat Commun 2020;11:3500.

15 Self WH, Tenforde MW, Stubblefield WB, et al. Seroprevalence of SARS-CoV-2 among frontline health care personnel in a multistate 
Hospital network - 13 academic medical centers, April-June 2020. MMWR Morb Mortal Wkly Rep 2020;69:1221-6.

16 Korth J, Wilde B, Dolff S, et al. SARS-CoV-2-specific antibody detection in healthcare workers in Germany with direct contact to COVID-19 patients. J Clin Virol 2020;128:104437.

17 Stubblefield WB, Talbot HK, Feldstein LR, et al. Seroprevalence of SARS-CoV-2 among frontline healthcare personnel during the first month of caring for patients with COVID-19-Nashville, Tennessee. Clin Infect Dis 2021;72:1645-8.

18 Rudberg A-S, Havervall S, Manberg A. SARS-CoV-2 exposure, symptoms and seroprevalence in health care workers. medRxiv 2020.

19 Steensels D, Oris E, Coninx L, et al. Hospital-Wide SARS-CoV-2 antibody screening in 3056 staff in a tertiary center in Belgium. JAMA 2020;324:195.
20 Moscola J, Sembajwe G, Jarrett M, et al. Prevalence of SARS-CoV-2 antibodies in health care personnel in the new York City area. JAMA 2020;324:893.

$21 \mathrm{He} \mathrm{J}$, Guo Y, Mao R, et al. Proportion of asymptomatic coronavirus disease 2019: a systematic review and meta-analysis. J Med Virol 2021;93:820-30

22 Bedford J, Enria D, Giesecke J, et al. COVID-19: towards controlling of a pandemic. Lancet 2020;395:1015-8.

23 Morcuende M, Guglielminotti J, Landau R. Anesthesiologists' and intensive care providers' exposure to COVID-19 infection in a new York City academic center: a prospective cohort study assessing symptoms and COVID-19 antibody testing. Anesth Analg 2020;131:669-76.

24 Seow J, Graham C, Merrick B, et al. Longitudinal observation and decline of neutralizing antibody responses in the three months following SARS-CoV-2 infection in humans. Nat Microbiol 2020;5:1598-607. 